\title{
Protein complexes identification based on go attributed network embedding
}

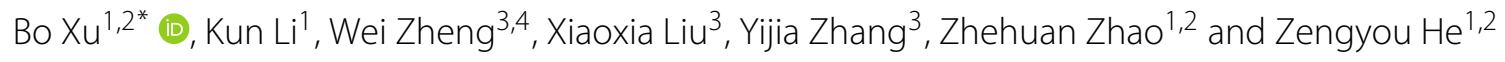

\begin{abstract}
Background: Identifying protein complexes from protein-protein interaction (PPI) network is one of the most important tasks in proteomics. Existing computational methods try to incorporate a variety of biological evidences to enhance the quality of predicted complexes. However, it is still a challenge to integrate different types of biological information into the complexes discovery process under a unified framework. Recently, attributed network embedding methods have be proved to be remarkably effective in generating vector representations for nodes in the network. In the transformed vector space, both the topological proximity and node attributed affinity between different nodes are preserved. Therefore, such attributed network embedding methods provide us a unified framework to integrate various biological evidences into the protein complexes identification process.

Results: In this article, we propose a new method called GANE to predict protein complexes based on Gene Ontology (GO) attributed network embedding. Firstly, it learns the vector representation for each protein from a GO attributed PPI network. Based on the pair-wise vector representation similarity, a weighted adjacency matrix is constructed. Secondly, it uses the clique mining method to generate candidate cores. Consequently, seed cores are obtained by ranking candidate cores based on their densities on the weighted adjacency matrix and removing redundant cores. For each seed core, its attachments are the proteins with correlation score that is larger than a given threshold. The combination of a seed core and its attachment proteins is reported as a predicted protein complex by the GANE algorithm. For performance evaluation, we compared GANE with six protein complex identification methods on five yeast PPI networks. Experimental results showes that GANE performs better than the competing algorithms in terms of different evaluation metrics.
\end{abstract}

Conclusions: GANE provides a framework that integrate many valuable and different biological information into the task of protein complex identification. The protein vector representation learned from our attributed PPI network can also be used in other tasks, such as PPI prediction and disease gene prediction.

Keywords: Protein complexes identification, Protein-protein interaction network, Network embedding

\section{Background}

With the advent of the post-genomic era, the focus of life science research has shifted from genomics to proteomics. One important task in proteomics is to detect protein complexes from protein-protein interaction (PPI) networks. The discovery of protein complexes is not only critical to reveal the principle of cellular organization and functions, but also helpful to predict protein functions,

\footnotetext{
*Correspondence: boxu@dlut.edu.cn

'School of Software Technology, Dalian University of Technology, No.321

Tuqiang Road, Economic Development Zone, 116024 Dalian, China

${ }^{2}$ Key Laboratory for Ubiquitous Network and Service Software of Liaoning,

116000 Dalian, China

Full list of author information is available at the end of the article
}

disease genes and drug-disease associations. With the advances of high-throughput technologies, many largescale PPI networks have been constructed [1,2]. Hence, it is highly demanding to develop effective computational methods for the accurate identification of novel protein complexes.

In recent years, many computational methods have been proposed to predict protein complexes from PPI networks. A PPI network is usually modeled as an undirected graph, where the nodes in the graph represent proteins and the edges represent the interactions between proteins. Roughly, most of these protein complexes identification methods are based on the principle that densely linked

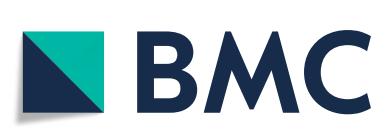

(C) The Author(s). 2018 Open Access This article is distributed under the terms of the Creative Commons Attribution 4.0 International License (http://creativecommons.org/licenses/by/4.0/), which permits unrestricted use, distribution, and reproduction in any medium, provided you give appropriate credit to the original author(s) and the source, provide a link to the Creative Commons license, and indicate if changes were made. The Creative Commons Public Domain Dedication waiver (http://creativecommons.org/publicdomain/zero/1.0/) applies to the data made available in this article, unless otherwise stated. 
regions in the PPI network correspond to actual protein complexes [3]. Therefore, the issue of predicting protein complexes can be formulated as the problem of detecting densely linked regions in PPI networks.

Existing computational methods for predicting protein complexes can be approximately divided into two broad categories [4]: (1) The methods based solely on PPI networks. These methods cluster the PPI network into multiple dense subnetworks only based on the topology of network [5]. They make use of merging, growing or partitioning strategies to detect protein complexes. Here, we just list a few typical methods in this category [6], e.g., CFinder [7], MCODE [8], LCMA [9], CMC [10], HACO [11], ClusterOne [12], MCL [13] and PEWCC [14]. (2) The methods based on PPI networks and some additional biological insights [15]. The biological insights are grouped as: core-attachment structure, evolutionary information, functional coherence, and mutually exclusive and co-operative interactions. CORE [16], $\mathrm{COACH}$ [17] and HUNTER [18] detected protein complexes based on the principle that each complex is composed by a core and its attachments. ProRank [19], ProRank+ [20] and the methods proposed by Sharan et al. [21, 22] detected conserved complexes across species based on the evolution of PPI networks. RNSC [23] and DECAFF [24] combined topological and GO information as functional information to detect complexes. Ozawa et al. [25] proposed a refinement method over MCODE and MCL to filter predicted complexes based on exclusive and co-operative interactions. Over the years, researchers tried to incorporate a variety of biological information to enhance the quality of predicted complexes [26]. However, it is still a challenge to integrate various biological evidences into a unified framework.

Recently, network embedding methods have shown to be effective in many graph data analysis tasks such as link prediction and network clustering [27]. Network embedding aims to represent nodes in the network in a lowdimensional space while preserving the node proximities. The definition of node proximities depends on the analytic tasks and application scenarios. According to the definition of node proximities, the state-of-the-art network embedding methods can be categorized into two groups: (1) structure-preserved network embedding; (2) attributed network embedding.

Structure-preserved network embedding methods focus on preserving the topological structure of the original network. Motivated by the similar power-law distribution of the vertices appearing in short random walk and the words in natural language, DeepWalk [28] regarded walks as the equivalent of sentences and then preserved the neighborhood structure of nodes by maximizing the co-occurrence probability between a target node and its context nodes within a truncated random walk window. Node2vec [29] proposed a method which can generate the neighborhoods of nodes using the 2nd order random walk. The LINE approach [30] solved the large-scale network embedding effectively by preserving the first and second order proximities.

Attributed network embedding targets at leveraging both the topological proximity and node/edge attribute affinity. MMDW [31] is a semi-supervised version of DeepWalk, which incorporates the labeling information into the network embedding. It jointly optimizes a maxmargin classifier and the representation learning model. By establishing the equivalent relationship between DeepWalk and matrix factorization, TADW [32] incorporates the rich text information into network embedding. AANE [33] is a scalable and efficient framework which learn a unified embedding representation by incorporating node attribute proximity into network embedding. It preserve the node proximity in both network structure space and attribute space.

The attributed network embedding method provides a general framework for incorporating both network structure information and additional node attribute information to generate a unified low-dimensional representation. This salient feature is particularly desirable in the context of protein complexes identification since using the additional biological information. The use of additional biological information sources often boost the identification performance significantly. Unfortunately, there are still no researches that exploit the attributed network embedding approach for protein complex detection. To fill this gap, we take the first attempt to investigate the feasibility and advantage of utilizing the attributed network embedding idea for protein complexes detection.

We propose a new method called GANE to predict protein complexes based on Gene Ontology(GO) attributed network embedding. The PPI network is represented as an attributed network in which the protein nodes are associated with GO slims. GANE first learns the vector representation for each protein from the GO attributed PPI network. Then, it uses a clique mining method to generate candidate cores. Consequently, a set of seed cores are generated from the set of candidate cores with density-based clique ranking and redundancy-based clique updating. For each seed core, its attachments are the proteins whose correlation score is larger than a threshold. The seed cliques with attachments are reported as the predicted protein complexes. In order to evaluate our method, we compared GANE with six classic protein complex identification methods, which are $\mathrm{COACH}$ [17], CMC [10], MCODE [8], ClusterOne [12], MCL [13] and PEWCC [14] on five different yeast PPI networks. Experiment results show that GANE performs better than the state-of-the-art methods with respect to different evaluation metrics. We summarize the contributions of this paper as follows: 
- To our knowledge, although some methods incorporate several biological information in different ways, our method is the first piece of work that incorporates the attributed network embedding idea into the protein complexes identification problem.

- Our method provides a framework that integrate many valuable biological information into the task of protein complex identification.

- The protein vector representation learned from our attributed PPI network can also be used in other tasks, such as PPI prediction and disease gene prediction.

The remainder of the paper is organized as follows. In "Methods" section, we present the GANE method. We compare GANE with six classic complex identification methods and show the experiment results in "Results and discussion" section. Finally, "Conclusions" section gives a conclusion of this paper.

\section{Methods}

The GANE method for protein complex prediction is a two-step procedure. Firstly, it learns the vector representation for each protein from the GO attributed PPI network. Based on the pair-wise vector representation similarity, a weighted adjacency matrix is constructed. Secondly, it uses a clique mining method to generate candidate cores. A set of seed cores are generated from the set of candidate cores with density-based clique ranking and redundancy-based clique updating. For each seed core, its attachments are the proteins whose correlation score is larger than a threshold. The seed cores with attachments are the predicted protein complexes. Figure 1 illustrates the basic pipeline of the GANE method in a vivid manner. Meanwhile, the major steps of our algorithm are presented in Table 1.

\section{Learning vector representations for proteins}

The network embedding technique transforms graphstructured data into vectorial data by learning the lowdimensional vector representation for each node in the network. Among existing network embedding methods, the AANE [33] aims at preserving both the topological similarity and node attribute similarity in the transformed space. Based on the AANE, we learn vector representations for proteins in the PPI network by preserving the proximities among proteins with respect to both the topological structure and GO attributes. The corresponding representation learning method is described below.

\section{Topological model}

In the GANE, a PPI network is represented as an undirected graph $G=(V, E)$, where the nodes in $V$ represent

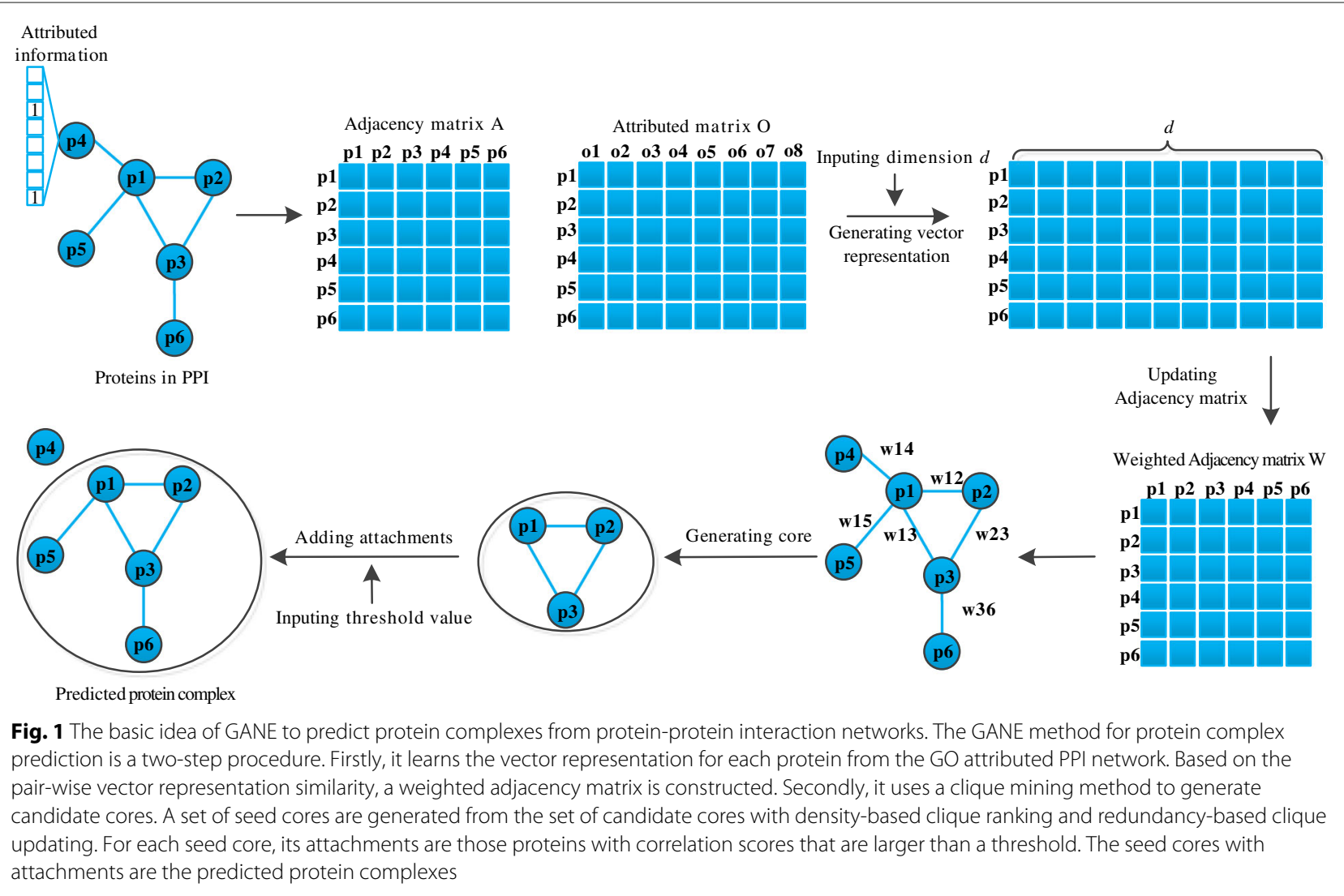


Table 1 Major steps of GANE

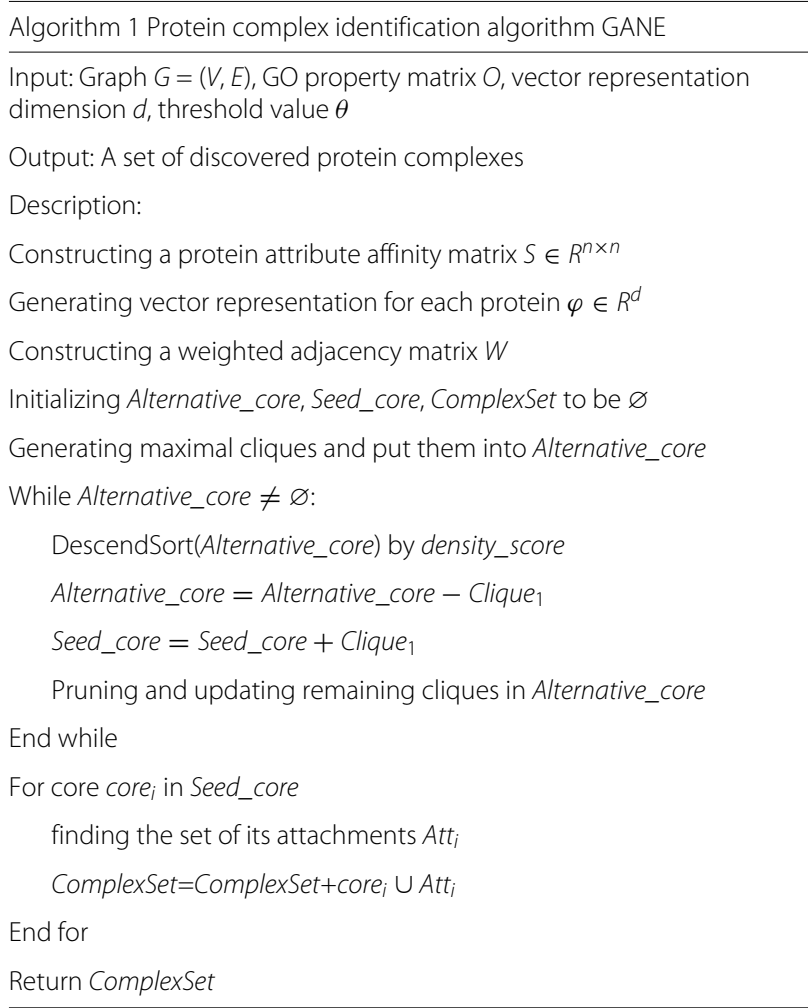

proteins and the edges in $E$ represent the interactions between proteins. In order to preserve the topological proximity between proteins in the original PPI network, a loss function is defined as:

$$
\ell_{1}=\sum_{i \in V} \sum_{j \in V} a_{i j}\left(\varphi_{i}-\varphi_{j}\right)^{2},
$$

where $\varphi_{i}$ and $\varphi_{j}$ are the vector representations of protein $i$ and protein $j$, the matrix $A \in R^{n \times n}$ represents the adjacency matrix of the PPI network, $a_{i j}=1$ only if there is an interaction between protein $i$ and protein $j$. Minimizing the penalty part $a_{i j}\left(\varphi_{j}-\varphi_{j}\right)^{2}$ means to minimize the embedding difference between $\varphi_{i}$ and $\varphi_{j}$ when $a_{i j}=1$ . Hence, proteins with similar topological structures will be forced to have similar vector representations. As a result, this model preserve the topological structures of the original PPI network.

\section{GO attributed model}

Gene Ontology (GO) is currently one of the most comprehensive ontology databases in the bioinformatics community [34]. It provides GO terms to describe three different aspects of gene product features: biological process $(B p)$, molecular function $(M f)$, and cellular component $(C c)$. GO slims is the cut-down version of GO, it contains a subset of the terms in the whole GO. They provide an overview on the ontology content without the details of the specific fine grained terms. GO slims give a comprehensive description on the biological attributes of proteins. Since GO slims of $C c$ include some protein complexes information, we only select GO slims of $B p$ and $M f$ as protein attributes.

After getting the attributed information for all the proteins in the PPI network, we generate an attribute matrix $O \in R^{n \times m}$, where $n$ represents the number of proteins and $m$ represents the number of GO slims attributes. Each entry $o_{i j}$ in the matrix $O$ describes whether protein $i$ has a corresponding GO slim $j$ or not with $o_{i j}=1$ or 0 . Based on the matrix $O$, we construct a protein attribute affinity matrix $S \in R^{n \times n}$. Each entry $s_{i j}$ is calculated as below:

$$
s_{i j}=\frac{\sum_{k=1}^{m} o_{i k} \times o_{j k}}{\sqrt{\sum_{k=1}^{m} o_{i k}^{2}} \times \sqrt{\sum_{k=1}^{m} o_{j k}^{2}}} .
$$

To preserve the proximity with respect to protein attributes, a loss function is defined as:

$$
\ell_{2}=\sum_{i \in V} \sum_{j \in V}\left(s_{i j}-\varphi_{i} \varphi_{j}^{T}\right)^{2},
$$

where $S \in R^{n \times n}$ is the protein attribute affinity matrix. Minimize this loss function means minimize the difference between the dot product of the vector representation $\varphi_{i}$ and $\varphi_{j}$ with the corresponding attribute similarity $s_{i j}$.

\section{Joint model for representation learning}

Since topological and biological properties are both important for protein complexes identification, we use the topological model and GO attributed model together to learn the representations of proteins. The final loss function is defined as:

$$
\ell=\sum_{i \in V} \sum_{j \in V} a_{i j}\left(\varphi_{i}-\varphi_{j}\right)^{2}+\lambda \sum_{i \in V} \sum_{j \in V}\left(s_{i j}-\varphi_{i} \varphi_{j}^{T}\right)^{2},
$$

where $\lambda$ is a parameter that controls the trade-off between topological and GO attributed properties. Since $\ell$ is separable for $\varphi_{i}$, the corresponding minimization problem can be reformulated as a bi-convex optimization problem. The original embedding problem is split into $2 n$ small convex optimization sub-problems. As shown in AANE [33], the distributed convex optimization technique ADMM $[35,36]$ can be used to solve this optimization problem. In each iteration, the $n$ updating steps of $\varphi_{i}$ is assigned to different workers in a distributed way. The distributed algorithm is guaranteed to converge to a local optimal point [35]. After solving the optimization problem of minimizing the loss function in Eq. (4), each protein is represented as a vector $\varphi \in R^{d}$, where $d$ represents the length of the embedding representation. 


\section{Weighted adjacency matrix}

After obtaining the vector representation of each protein $\varphi \in R^{d}$, we generate a weighted adjacency matrix $W \in$ $R^{n \times n}$ as below, where cos_sim is the function for calculating the cosine similarity between two connected proteins based on the embedding representations.

$$
w_{i j}=\left\{\begin{array}{ll}
\cos \_\operatorname{sim}\left(\varphi_{i}, \varphi_{j}\right) & a_{i j}=1 \\
0 & a_{i j}=0
\end{array} .\right.
$$

\section{Clustering based on core-attachment structure}

Gavin et al. [37] proposed that a protein complex is usually composed of two parts, a core and its attachments. Based on this principle, we detect protein complexes in two phases. Firstly, a set of seed cores are generated. Secondly, the attachments are included into each core based on their correlation strengths.

\section{Generating cores}

To generate cores, we use the cliques mining algorithm proposed by Tomita et al. [38] to enumerate all maximal cliques with at least three nodes in a PPI network. These cliques are considered as the candidate cores and we collected them into a Alternative_core set. Since not all the cliques in Alternative_core are suitable to be the cores of protein complexes, we prune the Alternative_core set to generate the Seed_core set based on the following procedure:

1 Cliques in Alternative_core are sorted in the descending order by density_score, denoted as Clique $_{1}$, Clique $_{2}, \ldots$, Clique . This density_score function considers both the inside connective density and biological correlation of each clique.

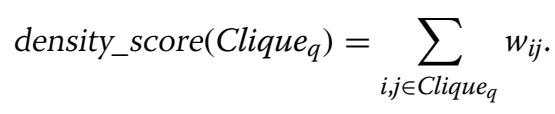

2 Remove Clique 1 from Alternative_core and put it into the Seed_core set.

3 For any other clique Clique $i \in$ Alternative_core that has an overlap with Clique $_{1}$, Clique $_{i}$ is updated with Clique $_{i}-$ Clique $_{1}$. After that, if $\mid$ Clique $_{i} \mid<3$, remove Clique $_{i}$ from Alternative_core.
This process repeats until Alternative_core is empty. The cliques in Seed_core are regarded as the core proteins in protein complexes.

\section{Adding attachments}

To detect attachments for each core, we focus on the strength of topological and biological connectivity between the core and the corresponding attachments. The correlation score between a clique in the Seed_core and a candidate attachment protein is calculated as below:

$$
\text { correlation_score }\left(p_{i}, \text { Clique }_{j}\right)=\frac{\sum_{k \in \text { Clique }_{j}} w_{i k}}{\mid \text { Clique }_{j} \mid},
$$

where protein $p_{i}$ is one of the neighbors of the corresponding core Clique $_{j}$. If the correlation score between protein $p_{i}$ and Clique $_{j}$ is larger than a threshold value $\theta$, $p_{i}$ is considered as one attachment of the corresponding clique.

Finally, each protein complex is generated by combining the core and its corresponding attachments.

\section{Results and discussion}

\section{Datasets}

Five yeast PPI networks were used in the performance comparison: DIP [39], Krogan-core [40], Krogan14k [40], Biogrid [41], Collins [42]. The detailed information of these five datasets are shown in Table 2 . The GO slim information was downloaded from the website https://downloads.yeastgenome.org/curation/ literature/go_slim_mapping.tab. To compare the predicted results with the reference complexes, we have constructed a standard complexes set by selecting all the protein complexes that had at least three proteins from MIPS, CYC2008, SGD, Aloy and TAP06. Consequently, there was a total 789 protein complexes in the reference set.

\section{Evaluation metrics}

To formally evaluate the performance of our method, we use the same evaluation metrics as other methods $[12,14]$.

Let $P$ denotes the set of predicted protein complexes from one method, the performance of this methods is mainly determined by the number of matched complexes

Table 2 The PPI data sets used in the experiment

\begin{tabular}{lllll}
\hline PPI networks & Number of proteins & Number of interactions & Average clustering coefficient & Average number of neighbors \\
\hline DIP & 4928 & 17,201 & 0.095 & 6.981 \\
Krogan-core & 2708 & 7123 & 0.188 & 5.261 \\
Krogan14k & 3581 & 14,076 & 0.122 & 7.861 \\
Biogrid & 5640 & 59,748 & 0.246 & 21.187 \\
Collins & 1622 & 9074 & 0.555 & 11.189 \\
\hline
\end{tabular}

Five yeast PPI networks were used in the performance comparison: DIP (Xenarios et al., 2002), Krogan-core (Krogan et al., 2006), Krogan14k (Krogan et al., 2006), Biogrid (Stark et al., 2006), Collins (Collins et al., 2007) 
between $P$ and the set of gold standard protein complexes $B$. To determine if a predicted protein complex $p \in P$ matches a known protein complex $b \in B$, we use the neighborhood affinity score $N A(p, b)$ defined in in Eq. (8):

$$
N A(p, b)=\frac{\left|V_{p} \cap V_{b}\right|^{2}}{\left|V_{p}\right| \times\left|V_{b}\right|},
$$

where $V_{p}$ is the set of proteins in the predicted protein complex $p$ and $V_{b}$ is the set of proteins in the reference protein complex $b$. Following the previous studies, $p$ and $b$ are considered to matched if $N A(p, b)$ is larger than 0.25 .

Based on the neighborhood affinity score, $N_{c p}$ is defined as the number of predicted complexes that match at least one real complex, and $N_{c b}$ is the number of real complexes that match at least one predicted complex.

$$
\begin{aligned}
& N_{c p}=|\{p \mid p \in P, \exists b \in B, N A(p, b) \geq \omega\}|, \\
& N_{c b}=|\{b \mid b \in B, \exists p \in P, N A(p, b) \geq \omega\}| .
\end{aligned}
$$

In Eqs. (9) and (10), $\omega$ is threshold parameter, which is typically specified to be 0.25 .

The first three measures used in the experiments for evaluating the performance of different methods are Precision, Recall and F-score. Precision is the proportion of predicted protein complexes that match at least one reference complex. Recall is the proportion of reference protein complexes that match at least one predicted complex. F-score is the harmonic mean of Precision and Recall.

$$
\begin{aligned}
& \text { Precision }=\frac{N_{c p}}{|P|}, \text { Recall }=\frac{N_{c b}}{|B|}, \\
& F-\text { score }=\frac{2 \times \text { Precision } \times \text { Recall }}{\text { Precision }+ \text { Recall }} .
\end{aligned}
$$

The other three metrics we used are clustering-wise sensitivity $(S n)$, clustering-wise positive predictive value $(P P V)$ and geometric accuracy $(A c c)$. Given $|B|$ reference complexes and $|P|$ predicted complexes, let $T_{i j}$ denote the number of proteins that are found both in reference complex $i$ and predicted complex $j$, and let $N_{i}$ denote the number of proteins in reference complex $i$. Then, $S n, P P V$, Acc are defined as follows:

$$
\begin{aligned}
& S n=\frac{\sum_{i=1}^{|B|} \max _{j=1}^{|P|}\left\{T_{i j}\right\}}{\sum_{i=1}^{|B|} N_{i}}, \\
& P P V=\frac{\sum_{j=1}^{|P|} \max _{i=1}^{|B|}\left\{T_{i j}\right\}}{\sum_{j=1}^{|P|} \sum_{i=1}^{|B|} T_{i j}}, \\
& A c c=\sqrt{S n \cdot P P V} .
\end{aligned}
$$

\section{Performance comparison}

For evaluating the performance of our algorithm, we compared our algorithm with six state-of-the-art protein complexes detection methods: $\mathrm{COACH}, \mathrm{CMC}, \mathrm{MCODE}$, ClusterOne , MCL and PEWCC. The parameters of these methods were set as the default values as mentioned in their original papers. The embedding dimension $d$, the harmonic value $\lambda$ and the threshold value $\theta$ of GANE were set to be $128,0.1$ and 0.3 respectively. For a fair comparison, we filtered out the complexes whose sizes are less than 3 in all algorithms. All experimental results were listed in Table 3 and Fig. 2.

As shown in Table 3, GANE achieved the highest Precision and F-score on four data sets: DIP, Krogancore, Krogan14k and Biogrid. It did not achieve the best Precision on the Collins dataset, but had the highest F-score. GANE did not achieve the highest Recall, probably because its number of predicted protein complexes is small. Overall, GANE performed the best with respect to the overall evaluation metric F-score for all datasets. In addition, our method reported the highest $A c c$ value on all datasets except for Krogan-core and Biogrid. For these two datasets, ClusterOne was the best with respect to Acc. The ClusterOne method detected protein complexes based on seeding and greedy growth. So, the protein complexes detected by ClusterOne generally had more proteins, and its Acc was higher than that of our method. But the Precision and F-score of Clusterone were all lower than our method.

In order to visually observe the comparative results, Fig. 2 showed the composition score $(F$-score $+A c c)$ of each method. In Fig. 2, the y-axis represented the sum of F-score and Acc. As shown in Fig. 2, our method always obtained the highest composition score. Therefore, our method outperformed other algorithms for all five datasets.

To examine the biological sense of the predicted protein complexes generated by GANE, we calculated the $P$-value by the tool GO::TermFinder [43]. Some of our unmatched predicted complexes actually had high biological significance. Due to the gold-standard complex set was still incomplete, these unmatched predicted complexes might be the new complexes that had not been discovered. Table 4 presented some case studies of GO analysis results from the DIP network. The min $P$-value represented the minimum $P$-value of the matched GO analysis results, it indicated that the collective occurrence of these proteins in a complex did not occur merely by chance. Thus, the predicted complex had a high probability to be real.

Expect GO slims, we also utilized gene expression profile as attribute information for complexes detection. The performances were shown in Additional file 1: Table S1.

\section{Parameter sensitivity}

In this part, we examined the sensitivity of GANE with respect to three parameters: the length of vector representation $d$, the harmonic value $\lambda$ and the threshold value $\theta$. 
Table 3 Performance comparison based on six evaluation metrics on the five yeast data

\begin{tabular}{|c|c|c|c|c|c|c|c|}
\hline Datasets & Methods & \#predicted complexes & \#matched complexes & Precision & Recall & F-score & Acc \\
\hline \multirow[t]{7}{*}{ DIP } & $\mathrm{COACH}$ & 570 & 263 & 0.450 & 0.620 & 0.521 & 0.243 \\
\hline & CMC & 179 & 108 & 0.603 & 0.394 & 0.477 & 0.219 \\
\hline & MCODE & 59 & 32 & 0.542 & 0.118 & 0.194 & 0.149 \\
\hline & ClusterOne & 341 & 133 & 0.390 & 0.343 & 0.365 & 0.227 \\
\hline & $\mathrm{MCL}$ & 451 & 69 & 0.153 & 0.172 & 0.162 & 0.190 \\
\hline & PEWCC & 666 & 413 & 0.620 & 0.469 & 0.534 & 0.230 \\
\hline & GANE & 324 & 202 & 0.623 & 0.550 & 0.584 & 0.254 \\
\hline \multirow[t]{7}{*}{ Krogan-core } & $\mathrm{COACH}$ & 348 & 206 & 0.592 & 0.460 & 0.518 & 0.217 \\
\hline & CMC & 128 & 86 & 0.672 & 0.304 & 0.419 & 0.206 \\
\hline & MCODE & 71 & 52 & 0.732 & 0.198 & 0.311 & 0.176 \\
\hline & ClusterOne & 522 & 190 & 0.364 & 0.464 & 0.408 & 0.273 \\
\hline & $\mathrm{MCL}$ & 376 & 126 & 0.335 & 0.414 & 0.371 & 0.262 \\
\hline & PEWCC & 630 & 425 & 0.675 & 0.406 & 0.507 & 0.214 \\
\hline & GANE & 208 & 161 & 0.774 & 0.436 & 0.558 & 0.229 \\
\hline \multirow[t]{7}{*}{ Krogan14k } & $\mathrm{COACH}$ & 570 & 263 & 0.461 & 0.465 & 0.463 & 0.217 \\
\hline & CMC & 396 & 187 & 0.472 & 0.440 & 0.455 & 0.210 \\
\hline & MCODE & 49 & 30 & 0.612 & 0.112 & 0.189 & 0.152 \\
\hline & ClusterOne & 225 & 105 & 0.467 & 0.302 & 0.366 & 0.222 \\
\hline & $\mathrm{MCL}$ & 445 & 133 & 0.299 & 0.323 & 0.311 & 0.233 \\
\hline & PEWCC & 934 & 500 & 0.535 & 0.418 & 0.470 & 0.217 \\
\hline & GANE & 247 & 169 & 0.684 & 0.442 & 0.537 & 0.234 \\
\hline \multirow[t]{7}{*}{ Biogrid } & $\mathrm{COACH}$ & 1507 & 469 & 0.311 & 0.657 & 0.422 & 0.276 \\
\hline & CMC & 1503 & 236 & 0.157 & 0.553 & 0.245 & 0.265 \\
\hline & MCODE & 58 & 16 & 0.276 & 0.043 & 0.075 & 0.181 \\
\hline & ClusterOne & 476 & 187 & 0.393 & 0.497 & 0.439 & 0.316 \\
\hline & $\mathrm{MCL}$ & 338 & 77 & 0.228 & 0.219 & 0.223 & 0.249 \\
\hline & PEWCC & 2781 & 1044 & 0.375 & 0.677 & 0.483 & 0.288 \\
\hline & GANE & 637 & 347 & 0.545 & 0.664 & 0.599 & 0.310 \\
\hline \multirow[t]{7}{*}{ Collins } & $\mathrm{COACH}$ & 251 & 188 & 0.749 & 0.522 & 0.615 & 0.280 \\
\hline & CMC & 153 & 104 & 0.680 & 0.390 & 0.496 & 0.255 \\
\hline & MCODE & 111 & 94 & 0.847 & 0.400 & 0.540 & 0.254 \\
\hline & ClusterOne & 195 & 143 & 0.733 & 0.511 & 0.602 & 0.290 \\
\hline & $\mathrm{MCL}$ & 183 & 134 & 0.732 & 0.506 & 0.598 & 0.286 \\
\hline & PEWCC & 570 & 477 & 0.837 & 0.426 & 0.564 & 0.252 \\
\hline & GANE & 199 & 163 & 0.819 & 0.491 & 0.615 & 0.293 \\
\hline
\end{tabular}

Both F-score and Acc are overall evaluation metrics, so the highest values of F-score and Acc are set in bold for each dataset

\section{Effect of the embedding vector dimension}

In the experiment, the embedding vector dimension $d$ was varied from 32 to 224 . Figure 3 a showed that the performance of our method was not very sensitive to the dimension parameter. Although the best results on different datasets were achieved with different dimension parameters, 128 was relatively a good choice in practice.

\section{Effect of the harmonic value}

The harmonic factor $\lambda$ balanced the contributions of topological and biological information for GANE. To investigate the impact of $\lambda$, we varied it from 0.00001 to 1000 . When $\lambda$ was relatively low, topological information contributed much to the performance of our method. With the increasing of $\lambda$, biological information contributed much. As shown in Fig. 3b, different datasets achieved 


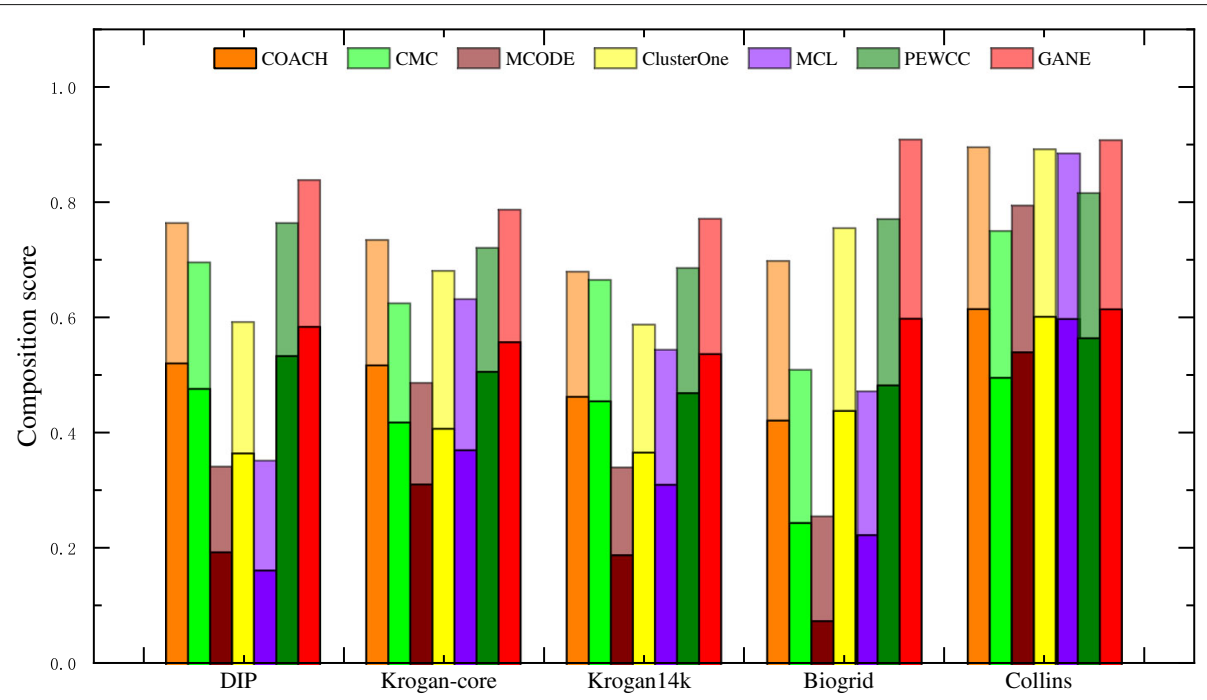

Fig. 2 Comparison with six protein complex detection algorithms in terms of the composite score of F-score and Acc. Shades of the same color indicate different evaluating scores. Each bar height reflects the value of the composite score

optimal solution with different $\lambda$. Here, we set $\lambda=0.1$ as default value.

\section{Effect of the threshold value}

The threshold value $\theta$ determined whether the neighbors of a core are included as its attachments. When the value $\theta$ was higher, it was harder for each neighbor to become an attachment. In other words, internal connections of the resulting protein complex were tighter. As shown in Fig. 3c, when $\theta$ was less than 0.1, the performance was relatively low. This was because when $\theta$ was small, most of the neighbors can be regarded as the corresponding attachments. The performance reached its peak when $\theta=0.3$, so we set 0.3 as its default value.

\section{Conclusions}

In this article, we propose an efficient method called GANE to detect protein complexes from PPI networks. GANE integrates biological evidences into the detecting process by learning vector representations for proteins from GO attributed network. As experimental results shown, GANE outperforms six protein complex detection methods on five different datasets. We concluded that the GO attributed network embedding can effectively enhance the quality of predicted complexes.

In the future, we will focus on investigating the following two questions:

1 How to learn the vector representations by incorporating more biological attributes in the PPI

Table 4 Examples of predicted complexes on the DIP dataset

\begin{tabular}{lllll}
\hline ID & Protein complex & Matched or not & Min P-value & GO-Description \\
\hline 1 & YLR376C YHL006C YIL132C YDR078C & No & $1.95 \mathrm{e}-10$ & DNA recombinase assembly \\
2 & YFR015C YJL137C YLR258W & No & $9.79 \mathrm{e}-07$ & Glycogen biosynthetic process \\
3 & YLR078C YLR026C YDR189W YDR498C & No & $1.42 \mathrm{e}-12$ & Vesicle fusion \\
& YLR268W YOR075W & & & Ceramide biosynthetic process \\
4 & YDR331W YMR298W YKL008C YHL003C & No & $3.96 \mathrm{e}-07$ & Ribosomal small subunit \\
& YGR060W & & & biogenesis \\
5 & YLR409C YER082C YKR060W YJR002W & No & & P.24e-23 \\
& YPR144C YER127W YNL132W YDR299W & & & endoplasmic reticulum \\
& YNL308C YCL059C YJL069C YCR057C & & 0.00014 & \\
& YDR324C YGR145W & & & \\
& YOR016C YHR140W YHL042W YBR106W & No & &
\end{tabular}




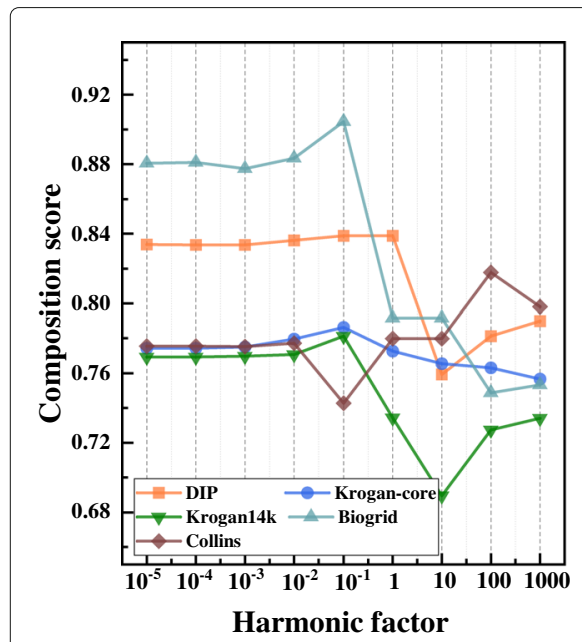

a

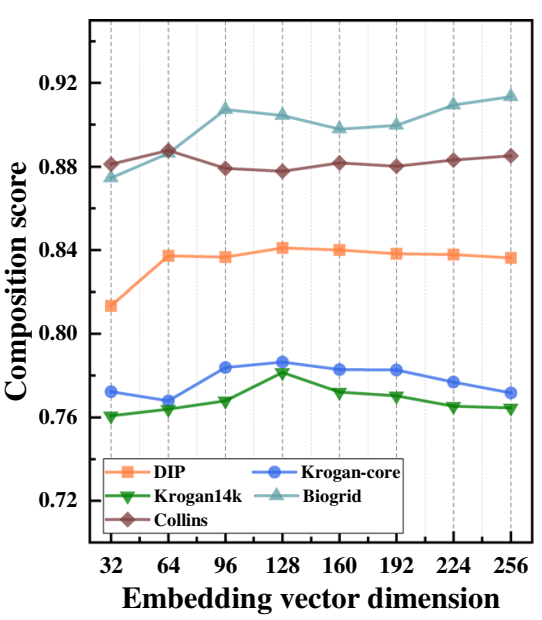

b

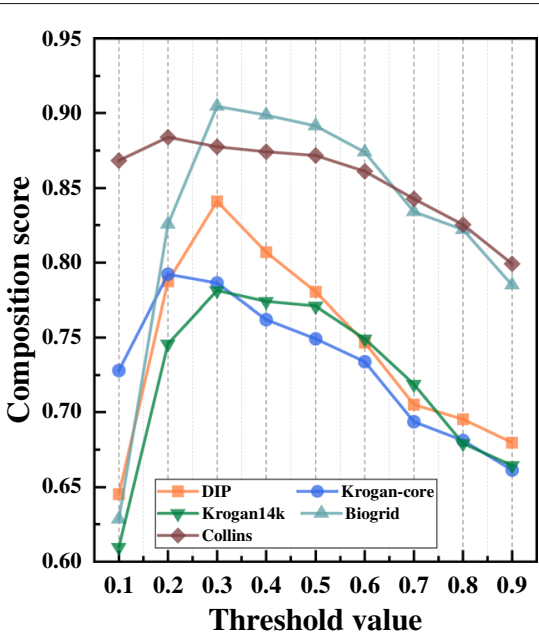

c

Fig. 3 The sensitivity of GANE with respect to three parameters. a The performance of GANE when embedding vector dimension $d$ was varied from 32 to 224. b The performance of GANE when harmonic value $\lambda$ was varied from 0.00001 to 1000. c The performance of GANE when threshold value $\theta$ was varied from 0.1 to 0.9

network? The incorporation of more biological evidences will further boost the identification performance.

2 How to apply the attributed network embedding methods to other biological networks, such as drug-drug interaction network and gene-phenotype network

\section{Additional file}

Additional file 1: Table S1. The performances of GANE with different attribute information. (DOCX $25 \mathrm{~kb}$ )

\section{Abbreviations}

Acc: Geometric accuracy; Bp: Biological process; Cc: Cellular component; GO:Gene Ontology; Mf: Molecular function; PPI: Protein-protein interaction; PPV: Clustering-wise positive predictive value; Sn: Clustering-wise sensitivity

\section{Acknowledgements}

The authors wish to thank Yu Liu and Peixu Gao for fixing grammatical errors and the reviewers for their helpful suggestions.

\section{Funding}

This work is supported by the grants from the Natural Science Foundation of China (No. 61502071 and 61572094) and the Fundamental Research Funds for the Central Universities (No. DUT18RC(3)004).

\section{Availability of data and materials}

All datasets and the source code of GANE are available at https://github.com/ LiKun-DLUT/GANE.

\section{Authors' contributions}

BX initiated and designed the study. WZ, XL, YZ and ZZ made substantial contributions to acquisition of data, analysis and interpretation of data. $\mathrm{KL}$ developed the algorithm and drafted the manuscript. BX and $\mathrm{ZH}$ involved in drafting the manuscript and revising it. All authors read and approved the final manuscript.
Ethics approval and consent to participate

Not applicable.

\section{Consent for publication}

Not applicable.

\section{Competing interests}

The authors declare that they have no competing interests.

\section{Publisher's Note}

Springer Nature remains neutral with regard to jurisdictional claims in published maps and institutional affiliations.

\section{Author details}

${ }^{1}$ School of Software Technology, Dalian University of Technology, No.321 Tuqiang Road, Economic Development Zone, 116024 Dalian, China. ${ }^{2}$ Key Laboratory for Ubiquitous Network and Service Software of Liaoning, 116000 Dalian, China. ${ }^{3}$ College of Computer Science and Technology, Dalian University of Technology, No.2 Linggong Road, Ganjingzi District, 116024 Dalian, China.

${ }^{4}$ College of software, Dalian JiaoTong University, 116000 Dalian, China.

Received: 22 May 2018 Accepted: 30 November 2018

Published online: 20 December 2018

\section{References}

1. Huttlin EL, Bruckner RJ, Paulo JA, Cannon JR, Ting L, Baltier K, et al. Architecture of the human interactome defines protein communities and disease networks. Nature. 2017;545(7655):505-9.

2. Wan C, Borgeson B, Phanse S, Tu F, Drew K, Clark G, et al. Panorama of ancient metazoan macromolecular complexes. Nature. 2015;525(7569): 339-44.

3. Wang J, Li M, Deng Y, Pan Y. Recent advances in clustering methods for protein interaction networks. BMC Genomics. 2010;11(3):S10.

4. Srihari S, Leong HW. A survey of computational methods for protein complex prediction from protein interaction networks. J Bioinforma Comput Biol. 2013;11(02):1230002.

5. Li X, Wu M, Kwoh CK, Ng SK. Computational approaches for detecting protein complexes from protein interaction networks: a survey. BMC Genomics. 2010;11(1):S3.

6. Bhowmick SS, Seah BS. Clustering and summarizing protein-protein interaction networks: A survey. IEEE Trans Knowl Data Eng. 2016;28(3): 638-58. 
7. Palla G. CFinder: locating cliques and overlapping modules in biological networks. Bioinformatics. 2006;22(8):1021-3.

8. Bader GD, Hogue CW. An automated method for finding molecular complexes in large protein interaction networks. BMC Bioinforma. 2003;4(1):2.

9. Li XL, Foo CS, Tan SH, Ng SK. Interaction graph mining for protein complexes using local clique merging. Genome Inform. 2005;16(2):260-9.

10. Liu G, Wong L, Chua HN. Complex discovery from weighted PPI networks. Bioinformatics. 2009;25(15):1891-7.

11. Wang H, Kakaradov B, Collins SR, Karotki L, Fiedler D, Shales M, et al. A Complex-based Reconstruction of the Saccharomyces cerevisiae Interactome. Mol Cell Proteome Mcp. 2009:8(6):1361.

12. Nepusz $T$, Yu H, Paccanaro A. Detecting overlapping protein complexes in protein-protein interaction networks. Nat Methods. 2012;9(5):471

13. Asur $S, U$ car D. Parthasarathy $S$. An ensemble framework for clustering protein-protein interaction networks. Bioinformatics. 2007;23(13):i29-i40.

14. Zaki, Nazar, Berengueres, Jose, Efimov, Dmitry. Protein complex detection using interaction reliability assessment and; weighted clustering coefficient. BMC Bioinforma. 2013;14(1):163.

15. Chen B, Fan W, Liu J, Wu FX. Identifying protein complexes and functional modules-from static PPI networks to dynamic PPI networks. Brief Bioinform. 2014;15(2):177.

16. Leung $\mathrm{HC}$, Xiang $\mathrm{Q}$, Yiu SM, Chin FY. Predicting protein complexes from PPI data: a core-attachment approach. J Comput Biol. 2009;16(2):133-44.

17. Wu M, Li X, Kwoh CK, Ng SK. A core-attachment based method to detect protein complexes in PPI networks. BMC Bioinforma. 2009;10(1):169.

18. Chin $\mathrm{CH}$, Chen SH, Ho CW, Ko MT, Lin CY. A hub-attachment based method to detect functional modules from confidence-scored protein interactions and expression profiles. BMC Bioinforma. 2010;11(Suppl 1): $1-9$.

19. Zaki N, Berengueres J, Efimov D. Detection of protein complexes using a protein ranking algorithm. Proteins Struct Funct Genet. 2012;80(10): 2459-68.

20. Hanna EM, Zaki N. Detecting protein complexes in protein interaction networks using a ranking algorithm with a refined merging procedure. BMC Bioinforma. 2014;15(1):1-11.

21. Sharan R, Ideker T, Kelley B, Shamir R, Karp RM. Identification of protein complexes by comparative analysis of yeast and bacterial protein interaction data. J Comput Biol J Comput Mol Cell Biol. 2005;12(6):835.

22. Hirsh E, Sharan R. Identification of conserved protein complexes based on a model of protein network evolution. Bioinformatics. 2007;23(2):e170-6.

23. King AD, Przulj N, Jurisica I. Protein complex prediction via cost-based clustering. Bioinformatics. 2004:20(17):3013-20.

24. Li XL, Foo CS, Ng SK. Discovering protein complexes in dense reliable neighborhoods of protein interaction networks. In: Computational Systems Bioinformatics. 2007. p. 157

25. Ozawa $Y$, Saito R, Fujimori S, Kashima H, Ishizaka M, Yanagawa $H$, et al. Protein complex prediction via verifying and reconstructing the topology of domain-domain interactions. BMC Bioinforma. 2010;11(1):1-12.

26. Ji J, Zhang A, Liu C, Quan X, Liu Z. Survey: Functional Module Detection from Protein-Protein Interaction Networks. IEEE Trans Knowl Data Eng. 2013;26(2):261-77.

27. Cui P, Wang X, Pei J, Zhu W. A Survey on Network Embedding; 2017. arXiv preprint arXiv:171108752.

28. Perozzi B, Al-Rfou R, Skiena S. Deepwalk: Online learning of social representations. In: Proceedings of the 20th ACM SIGKDD international conference on Knowledge discovery and data mining. New York: ACM; 2014. p. 701-10.

29. Grover A, Leskovec J. node2vec: Scalable feature learning for networks. In: Proceedings of the 22nd ACM SIGKDD international conference on Knowledge discovery and data mining. New York: ACM; 2016. p. 855-64.

30. Tang J, Qu M, Wang M, Zhang M, Yan J, Mei Q. Line: Large-scale information network embedding. In: Proceedings of the 24th International Conference on World Wide Web. International World Wide Web Conferences Steering Committee. 2015. p. 1067-77.

31. Tu C, Zhang W, Liu Z, Sun M. Max-Margin DeepWalk: Discriminative Learning of Network Representation. In: IJCAI. 2016. p. 3889-95.

32. Yang C, Liu Z, Zhao D, Sun M, Chang EY. Network Representation Learning with Rich Text Information. In: IJCAI. 2015. p. 2111-7.
33. Huang X, Li J, Hu X. Accelerated attributed network embedding. In: Proceedings of the 2017 SIAM International Conference on Data Mining SIAM; 2017. p. 633-41.

34. Consortium GO. The gene ontology (GO) project in 2006. Nucleic Acids Res. 2006;34(suppl_1):D322-6.

35. Boyd S, Parikh N, Chu E, Peleato B, Eckstein J, et al. Distributed optimization and statistical learning via the alternating direction method of multipliers. Found Trends ${ }^{\oplus}$ Mach Learn. 2011;3(1):1-122.

36. Hallac D, Leskovec J, Boyd S. Network lasso: Clustering and optimization in large graphs. In: Proceedings of the 21th ACM SIGKDD international conference on knowledge discovery and data mining. New York: ACM; 2015. p. 387-96.

37. Gavin AC, Aloy P, Grandi P, Krause R, Boesche M, Marzioch M, et al. Proteome survey reveals modularity of the yeast cell machinery. Nature. 2006;440(7084):631.

38. Tomita E, Tanaka A, Takahashi H. The worst-case time complexity for generating all maximal cliques and computational experiments. Theor Comput Sci. 2006;363(1):28-42.

39. Xenarios I, Salwinski L, Duan XJ, Higney P, Kim SM, Eisenberg D. DIP, the Database of Interacting Proteins: a research tool for studying cellular networks of protein interactions. Nucleic Acids Res. 2002;30(1):303-5.

40. Krogan NJ, Cagney G, Yu H, Zhong G, Guo X, Ignatchenko A, et al. Global landscape of protein complexes in the yeast Saccharomyces cerevisiae. Nature. 2006;440(7084):637

41. Stark C, Breitkreutz BJ, Reguly T, Boucher L, Breitkreutz A, Tyers M. BioGRID: a general repository for interaction datasets. Nucleic Acids Res. 2006;34(suppl_1):D535-9.

42. Collins SR, Kemmeren P, Zhao XC, Greenblatt JF, Spencer F, Holstege FC, et al. Toward a comprehensive atlas of the physical interactome of Saccharomyces cerevisiae. Mol Cell Proteomics. 2007;6(3):439-50.

43. Boyle El, Weng S, Gollub J, Jin H, Botstein D, Cherry JM, et al. GO:: TermFinder-open source software for accessing Gene Ontology information and finding significantly enriched Gene Ontology terms associated with a list of genes. Bioinformatics. 2004;20(18):3710-5.

\section{Ready to submit your research? Choose BMC and benefit from}

- fast, convenient online submission

- thorough peer review by experienced researchers in your field

- rapid publication on acceptance

- support for research data, including large and complex data types

- gold Open Access which fosters wider collaboration and increased citations

- maximum visibility for your research: over $100 \mathrm{M}$ website views per year

At $\mathrm{BMC}$, research is always in progress.

Learn more biomedcentral.com/submissions 